
\title{
25 Research Soure \\ Effect of Levosimendan on the Prognosis of Cardiac Surgery in Patients with Cardiac Insufficiency
}

\section{Heya $\mathrm{Na}$}

The Second Affiliated Hospital of Zhengzhou University

\section{Xianen Fa ( $\nabla$ faxianennhy@163.com )}

The Second Affiliated Hospital of Zhengzhou University

\section{Yuyang Zhou}

The Second Affiliated Hospital of Zhengzhou University

\section{Xiangyang Li}

The Second Affiliated Hospital of Zhengzhou University

\section{Rui Zhu}

The Second Affiliated Hospital of Zhengzhou University

\section{Lei Liu}

The Second Affiliated Hospital of Zhengzhou University

\section{Bing Liu}

The Second Affiliated Hospital of Zhengzhou University

\section{Research article}

Keywords: levosimendan, cardiac operative, propensity score matching

Posted Date: October 16th, 2020

DOI: https://doi.org/10.21203/rs.3.rs-92126/v1

License: (c) (1) This work is licensed under a Creative Commons Attribution 4.0 International License.

Read Full License 


\section{Abstract}

Objective: To retrospectively analyze the effect of levosimendan on the survival and prognosis of cardiac surgery patients with LVEF $<40 \%$.

Methods: the clinical data of 224 patients with preoperative LVEF $<40 \%$ were retrospectively analyzed. According to different treatment schemes, the patients were divided into levosimendan group $(n=60)$ and no-levosimendan group $(n=164$,). The control group was treated with routine treatment, and the observation group was treated with levosimendan on the basis of routine treatment. Then a multivariate logistic regression model with a propensity score analysis was used to limit biases and finally the data of 40 patients in each group were selected for analysis.

Results: Hemodynamic data showed that the cardiac index, LVEF and PAOP of patients in levosimendan group were significantly improved. The concentration of serum lactic acid in the levosimendan group was lower than that in the control group $(P<0.05)$. At the same time, postoperative ICU and hospital stay were significantly reduced in levosimendan group $(P<0.05$.), Logistics regression analysis showed that levosimendan was the only protective factor for Low cardiac output syndrome (LCOS) (HR=4.33; 95\% confidence interval: $1.27-14.78 ; \mathrm{P}=0.019$ ).

Conclusion: levosimendan can better improve hemodynamics and reduce postoperative ICU time and hospital stay. The use of it tended to decrease the incidence of LCOS significantly.

\section{Introduction}

Over the last few decades, mortality associated with heart surgery has declined significantly, despite the increased complexity of the procedure. Although the average perioperative mortality rate is currently 1$2 \%$, the incidence of major cardiovascular complications remains high ${ }^{1}$.

Low cardiac output syndrome (LCOS) is the most common and serious complication associated with increased morbidity, short-and long-term mortality, and increased utilization of health-care resources. In addition, the mortality rate of LCOS patients after heart surgery can exceed $20 \%$. The management of LCOS varies with its causes and manifestations. Recognition and treatment of precipitating factors are of prominent. $^{2}$

Levosimendan, as a calcium-sensitizing activity, is associated with positive inotropic action and increased myocardial contractility. However, compared with other inotropes, it did not increase intracellular calcium or cyclic adenosine. Thus, the absence of an associated increase in myocardial oxygen consumption also appears to be particularly beneficial for patients undergoing cardiac surgery with limited cardiac reserve. ${ }^{3}$

This agent has been used in clinical practice since 2000, especially for the care of patients with heart failure ${ }^{4}$. However, the evidence for its use in surgery is scant. Therefore, we intend to retrospectively 
analyze the specific efficacy of levosimendan in patients with a left ventricular ejection fraction (LVEF) less than $40 \%$ after cardiac surgery.

\section{Material And Methods}

This study was conducted with approval from the Ethics Committee of The Second Affiliated Hospital of Zhengzhou University (approval number 2018-019). As the nature of observational and retrospective study, informed consent was waived.

\section{Selection of the study sample}

This retrospective cohort study was conducted in the Cardiac surgery department of the Second Affiliated Hospital of Zhengzhou University from January 2017 to June 2020.

We target the recruitment of patients aged over 18 , scheduled for isolated CABG, isolated valve, or combined CABG/valve surgery with cardiopulmonary bypass, who have a LVEF less than $40 \%$, regardless of the method of assessment. All patients were consecutively evaluated. Exclusion criteria were: age $<18$ years, preoperative renal failure (creatinine clearance $<30 \mathrm{ml} / \mathrm{min}$ ), liver failure (prothrombine time $<50 \%$, in the absence of vitamin K antagonist) Pregnancy, emergency surgery, defined as surgery within the $24 \mathrm{~h}$ of the operative indication.

During the study period, give the attending physician full discretion to administer any other medication, including levosimendan and other vasoactive drugs, as appropriate. Levosimendan was administered without a bolus, by continuous infusion at $0.2 \mathrm{ug} / \mathrm{kg} / \mathrm{min}$ for $24 \mathrm{~h}$.

\section{Data collection}

Data were collected on: age; gender; body mass index $>24 \mathrm{~kg} / \mathrm{m}^{2}$; hypertension; diabetes mellitus; cardiopulmonary bypass time; aortic cross-clamping and serum lactic acid levels.

Echocardiography was performed by the same team with three ultrasound operators at baseline, intraoperative and postoperative of each patient. The LVEF was measured using biplane Simpson's method from the two-dimensional echocardiography.

All patients underwent continuous monitoring of heart rate and measurement of hemodynamic parameters including pulmonary artery occlusion pressure (PAOP) and cardiac index.

Time 0 was defined as the initial hemodynamic measurement, time 1 was recorded on arrival back in the cardiovascular ICU following surgery. Times 2 to 3 were $12 \mathrm{~h}$ and $24 \mathrm{~h}$ after arrival in the cardiovascular ICU following surgery, respectively.

Patients underwent 90 days of postsurgical follow-up, with survival status during this period being recorded. 


\section{Clinical definitions and study endpoints}

LCOS incidence was the primary outcome of this trial. LCOS was defined as the presence of low cardiac index $\left(<2.2 \mathrm{~L} / \mathrm{min} / \mathrm{m}^{2}\right)^{2}$, elevated PAOP $(>16 \mathrm{mmHg})$ and a SO $\mathrm{SO}^{2}<60 \%$. Secondary outcomes for this trial included (a) durations of ICU and hospital stay; (b) serum lactic acid levels at 0,12 , and $48 \mathrm{~h}$ post-surgery; (c) invasive hemodynamic parameters at 0,12 , and $48 \mathrm{~h}$ post-surgery.

\section{Statistical analysis}

Results were expressed as frequencies and percentages for categorical variables, and as means and standard deviations for continuous variables. Prior to propensity score matching, continuous variables were compared using Student's t test or the Mann Whitney test, as appropriate. Qualitative variables were compared using Pearson s Chi-square or Fisher s exact test, as appropriate. Mortality at 90 days after cardiac surgery was estimated using the Kaplan Meier method and compared using the log-rank tests. A two-sided $p<0.05$ was the significance threshold.

The propensity score was defined as the probability of exposure to levosimendan. In order to limit over adjustment due to the use of this score ${ }^{5}$, we selected only the covariates most likely to introduce a confounding bias $^{6}$. The propensity score was estimated using a logistic regression adjusted for age, sex, body mass index $>24 \mathrm{~kg} / \mathrm{m}^{2}$, presence of high blood pressure and diabetes. Matching was then performed between one patient exposed to levosimendan and up to one unexposed patients ${ }^{7}$, with a propensity score caliper of 0.05 .

After propensity score matching, Baseline demographic, clinical and echocardiographic data were estimated again to compare baseline characteristics and to therefore assess the accuracy of the matching procedure.

Associations between baseline factors and LCOS incidence were assessed using univariate analysis and multivariate logistic regression modeling. Hazard ratios (HR) and their $95 \%$ confidence intervals were calculated. A two-tailed $p$ value $<0.05$ was considered significant. All the statistical analyses were performed at the Second Affiliated Hospital of Zhengzhou University (Henan Province, China) with the use of SPSS software, version 25.0.

\section{Results}

\section{Study population}

Over the study period, 271 patients in high-risk with an ejection fraction less than $40 \%$ were included in the study. Among these, 47 patients were excluded from the study ( 12 were $<18$ years, 6 had preoperative renal failure, 3 had liver failure, 6 were pregnant, 20 received emergency surgery). A total of 224 patients with severely reduced left ventricular ejection fraction (LVEF $<40 \%$ ) were eligible for the study. In 20172018, in 2018-2019, in 2019-2020 and in 2020-2021, seven out of 35 patients (20\%), 20 out of 63 
(31.7\%), 32 out 75 (42.7\%) and 11 out 51 (21.6\%) were, respectively, treated with levosimendan. Forty propensity-matched patients were evaluated in the levosimendan group and 40 in the non-levosimendan group (Fig. 1).

\section{Characteristics and outcome of the 224 pre-matched patients}

Table 1 presents the basic characteristics of the included 224 patients (prior to matching). The average age was $55.29 \pm 8$ years and the proportion of male was $47.3 \%$. The proportion of body mass index greater than $24 \mathrm{~kg} / \mathrm{m}^{2}$ accounted for $48.7 \%$ (Table 1). During the study period, 60 patients were treated with levosimendan (26.8\%). There was no difference in mean cardiopulmonary bypass time and aortic crossclamp time compared with the control group. All the patients in the levosimendan group and the nolevosimendan group had severely reduced left ventricular ejection fraction with LVEF $<40 \%$, but the difference between the groups was statistically significant $(P=0.02)$.

\section{Characteristics and outcome of the $\mathbf{8 0}$ matched patients}

After propensity score matching, differences in the characteristics of patients between the levosimendan group and the non-levosimendan group were not statistically significant (Table 2).

Baseline hemodynamic parameters and serum lactate concentration were comparable in both groups (Fig. 2). Hemodynamic data demonstrate a rapid improvement in cardiac index, LVEF and PAOP in patients receiving levosimendan. Serum lactate concentration was consistently lower in levosimendan group at majority of time intervals. These effects persisted at all time intervals during and after surgery. Significant reduction in ICU and hospital stay occurred in levosimendan group at the postoperative period as compared to no levosimendan group $(P<0.05)$.

Out of 80 patients, 63 patients $(78.75 \%)$ were not presented in the postoperative phase with low cardiac output syndrome (LCOS). After propensity score matching, exposure to levosimendan was the only remaining factor associated with a significant reduction in the occurrence of LCOS rates (hazard ratio $=$ 4.33; $95 \%$ confidence interval: $1.27-14.78 ; P=0.019)$ (Table 3$)$.

\section{Discussion}

This retrospective study suggests that patients with a severely reduced left ventricular ejection fraction due to impaired contractility may probably benefit from levosimendan.

This agent acutely improves hemodynamics during postoperative recovery and also reduced the length of ICU stay and the length of hospital stay.

As far as we known, levosimendan's unique properties are attributed to its multiple and complementary mechanisms of action. 
In addition to its hemodynamic actions on coronary and pulmonary vessels, another hypothesis for the beneficial effects of levosimendan may be its positive effects on systemic vasodilation ${ }^{3}$. This is relevant to the reduction on serum lactic acid. Bravo et al conducted a study in the critically ill infants with congenital heart defects suffering from LCOS and found that many poor perfusion physiological variables and biomarkers indicators showed a different profile after levosimendan infusion, such as a significant decrease in serum lactate levels. Finally, levosimendan served a beneficial function on cerebral and systemic perfusion in this restricted patients ${ }^{8}$.

In addition, the patients with severe, low-output heart failure were also able to benefit from levosimendan ${ }^{9}$ ${ }^{10}$. In our view, extending the study population to non-cardiac surgery patients is relevant for physicians in the field because low cardiac output syndrome may occur in many cases.

To our knowledge, two other randomized controlled trials (RCTs) have not shown beneficial effects of the use of levosimendan compared with placebo in patients with preoperative severely depressed LVEF ${ }^{11} 12$. And Wang et al. also suggested that levosimendan had no significance for the improvement of the incidence of postoperative $\operatorname{LCOS}^{13}$. The beneficial effects of levosimendan are still debated in clinical practice. Nonetheless, researchers in the first study reported a beneficial effect in a subgroup of patients with a more severe reduction of $\operatorname{LVEF}^{12}$. Another analysis also shows that the beneficial effect of levosimendan can only be observed in patients with LVEF $<50 \%{ }^{14}$.

Van et al., divided the patients into different cardiac surgery methods ${ }^{15}$. Desai et al studied patients undergoing off-pump coronary artery bypass surgery ${ }^{16}$. In terms of the incidence of LCOS, no significant results were observed in either study, which is consistent with our observation. A large mate analysis also suggested that levosimendan could benefit the patients with lower LVEF $(<35 \%)$ in terms of the incidence of postoperative LCOS ${ }^{17}$. Levosimendan can reduce the incidence of LCOS after heart surgery, which can only be observed in patients with a more severe reduction of LVEF ${ }^{18}$. All of our patients had significant hemodynamic disorders with an ejection fraction less than $40 \%$.

In addition, Orriach et al. found that levosimendan can improve the renal function of postoperative LCOS patients ${ }^{19}$. This suggests that levosimendan can not only reduce the incidence of LCOS, but also benefit the patients who have already experienced LCOS, expanding the scope of application of levosimendan to a certain extent.

\section{Limitations}

Our study has several limitations. The retrospective nature of the analysis is clearly a weakness. In addition, our study may suffer from a lack of power because of the low number of patients and events evaluated. The main limitation of our study is that administration of levosimendan was not randomized. However, to limit biases due to the absence of randomization, we used a multivariate logistic regression model with a propensity score analysis. 


\section{Conclusion}

This study suggests that levosimendan can better improve hemodynamics and reduce postoperative ICU time and hospital stay. And the use of levosimendan tended to decrease the incidence of LCOS significantly.

\section{Abbreviations}

LCOS: Iow cardiac output syndrome; LVEF: left ventricular ejection fraction; ICU: intensive care unit.

\section{Declarations}

\section{Ethical Approval and Consent to participate}

This study was conducted with approval from the Ethics Committee of The Second Affiliated Hospital of Zhengzhou University (approval number 2018-019). The need for informed consent was waived because of the observational and retrospective nature of the study.

\section{Consent for publication}

Not applicable.

\section{Availability of supporting data}

The data used and/or analyzed in the present study are available from the corresponding author on reasonable request.

\section{Competing interests}

The authors declare that they have no competing interests.

\section{Funding}

This study is funded by National Science and Technology Planning Project of China, 2011BAl11B21 and Innovative talents of health science and technology in Henan Province of China, 201003040.

\section{Authors' contributions}

$\mathrm{HN}$ and XF had full access to all of the data in the study and take responsibility for the integrity of the data and the accuracy of the data analysis. $\mathrm{HN}, \mathrm{XF}$ and $\mathrm{YZ}$ conceptualized and designed the study. $\mathrm{HN}$, $\mathrm{XL}, \mathrm{RZ}$ and $\mathrm{BL}$ were involved in acquisition of data. $\mathrm{HN}, \mathrm{XL}, \mathrm{RZ}, \mathrm{LL}$ and $\mathrm{BL}$ analyzed and interpreted the data. $\mathrm{HN}$ and $\mathrm{BL}$ drafted the manuscript. $X \mathrm{~F}, \mathrm{YZ}$ and $\mathrm{LL}$ critically revised the manuscript for important intellectual content. $\mathrm{HN}$ and $\mathrm{XL}$ carried out the statistical analysis. $\mathrm{XF}$ and $\mathrm{YZ}$ were involved in funding, 
administrative, technical or material support. XF, YZ, XL, RZ, LL and BL supervised the study. All authors read and approved the final manuscript.

\section{Acknowledgements}

Not applicable.

\section{References}

1. Lomivorotov VV, Efremov SM, Kirov MY, et al. Low-Cardiac-Output Syndrome After Cardiac Surgery. 2016:S1053077016301513.

2. Follath $\mathrm{F}$, Cleland $\mathrm{J}$, Just $\mathrm{H}$, et al. Efficacy and safety of intravenous levosimendan compared with dobutamine in severe low-output heart failure (the LIDO study): a randomised double-blind trial. 2002;360(9328):196-202. doi: 10.1016/s0140-6736(02)09455-2

3. Levin R, Degrange M, Del Mazo C, et al. Preoperative levosimendan decreases mortality and the development of low cardiac output in high-risk patients with severe left ventricular dysfunction undergoing coronary artery bypass grafting with cardiopulmonary bypass. 2012;17(3):125-30.

4. Papp Z, Agostoni P, Alvarez J, et al. Levosimendan Efficacy and Safety: 20 Years of SIMDAX in Clinical Use. 2020;76(1):4-22. doi: 10.1097/fjc.0000000000000859

5. Schuster T, Lowe W, Platt RJJoce. Propensity score model overfitting led to inflated variance of estimated odds ratios. 2016;80:97-106. doi: 10.1016/j.jclinepi.2016.05.017

6. Brookhart MA, Schneeweiss S, Rothman KJ, et al. Variable selection for propensity score models. 2006;163(12):1149-56.

7. Austin PCJMBR. An Introduction to Propensity Score Methods for Reducing the Effects of Confounding in Observational Studies. 2011;46(3):399-424.

8. Bravo MC, Lopez P, Cabanas F, et al. Acute Effects of Levosimendan on Cerebral and Systemic Perfusion and Oxygenation in Newborns: An Observational Study. 2011;99(3):217-23.

9. Cholley B, Levy B, Fellahi J, et al. Levosimendan in the light of the results of the recent randomized controlled trials: an expert opinion paper. 2019;23(1):385. doi: 10.1186/s13054-019-2674-4

10. Cosentino N, Niccoli G, Fracassi F, et al. Rationale, Experimental Data, and Emerging Clinical Evidence on Early and Preventive Use of Levosimendan in Patients with Ventricular Dysfunction. 2019 doi: 10.1093/ehjcvp/pvz065

11. Landoni G, Lomivorotov V, Alvaro G, et al. Levosimendan for Hemodynamic Support after Cardiac Surgery. 2017;376(21):2021-31. doi: 10.1056/NEJMoa1616325

12. Mehta R, Leimberger J, van Diepen S, et al. Levosimendan in Patients with Left Ventricular Dysfunction Undergoing Cardiac Surgery. 2017;376(21):2032-42. doi: 10.1056/NEJMoa1616218

13. Wang A, Cui C, Fan Y, et al. Prophylactic use of levosimendan in pediatric patients undergoing cardiac surgery: a prospective randomized controlled trial. 2019;23(1):428. doi: 10.1186/s13054-0192704-2 
14. Qiang H, Luo X, Huo J, et al. Perioperative Use of Levosimendan Improves Clinical Outcomes in Patients After Cardiac Surgery: A Systematic Review and Meta-Analysis. 2018;72(1):11-18.

15. Van Diepen S, Mehta RH, Leimberger JD, et al. Levosimendan in patients with reduced left ventricular function undergoing isolated coronary or valve surgery. 2019

16. Desai P, Sarkar M, Umbarkar SJAoCA. Prophylactic preoperative levosimendan for off-pump coronary artery bypass grafting in patients with left ventricular dysfunction: Single-centered randomized prospective study. 2018;21(2):123-28.

17. Weber C, Esser M, Eghbalzadeh K, et al. Levosimendan Reduces Mortality and Low Cardiac Output Syndrome in Cardiac Surgery. 2020;68(5):401-09. doi: 10.1055/s-0039-3400496

18. Weber C, Esser M, Eghbalzadeh K, et al. Levosimendan Reduces Mortality and Low Cardiac Output Syndrome in Cardiac Surgery. 2019

19. Orriach JLG, Arce IN, Rodriguez PH, et al. Preservation of renal function in cardiac surgery patients with low cardiac output syndrome: levosimendan vs beta agonists. 2019;19(1):212.

\section{Tables}

Table 1

Baseline patient characteristics in pre-matched groups

\begin{tabular}{|lllll|}
\hline Variables & Total & Levosimendan & P value \\
\hline Male & $(\mathbf{n = 2 2 5 )}$ & No(n=164) & Yes(n=60) & \\
\hline Age (years) & $106(47.3)$ & $76(46.3)$ & $30(50)$ & 0.627 \\
\hline BMI $₫ 24 \mathrm{~kg} / \mathrm{m}^{2}$ & $55.29 \pm 8.360$ & $52.60 \pm 7.877$ & $62.63 \pm 4.214$ & 0.000 \\
\hline Isolated CABG & $109(48.7)$ & $75(45.7)$ & $34(56.7)$ & 0.147 \\
\hline Isolated valve surgery & $70(31.3)$ & $52(31.7)$ & $18(30)$ & 0.951 \\
\hline Combined CABG and valve & $86(38.4)$ & $62(37.8)$ & $24(40)$ & \\
\hline Diabetes mellitus & $68(30.4)$ & $50(30.5)$ & $18(30)$ & 0.211 \\
\hline Hypertension & $93(41.5)$ & $64(39.0)$ & $29(48.3)$ & 0.901 \\
\hline CPB(min) & $103(46.0)$ & $75(45.7)$ & $28(46.7)$ & 0.235 \\
\hline Aortic crossclamp time(min) & $96.83 \pm 16.219$ & $97.62 \pm 16.129$ & $94.68 \pm 16.406$ & 0.231 \\
\hline LVEF(\%) & $35.49 \pm 3.131$ & $35.20 \pm 3.216$ & $36.30 \pm 2.751$ & 0.012 \\
\hline Results are expressed as mean \pm SD or number (\%) & & & \\
\hline $\begin{array}{l}\text { CABG, Coronary artery bypass grafting; BMl, body mass index; LVEF, left ventricular ejection fraction; } \\
\text { CPB, cardiopulmonary bypass }\end{array}$ & & & \\
\hline
\end{tabular}


Table 2

Patient characteristics in propensity-matched groups

\begin{tabular}{|lllll|}
\hline Variables & Total & Levosimendan & & P \\
value
\end{tabular}


Table 3

Baseline factors predictive of LCOS incidence

\begin{tabular}{|llll|}
\hline Variables & LCOS & & P value \\
\hline Male & No(n=63) & Yes(n=17) & \\
\hline BMI $₫ 24 \mathrm{~kg} / \mathrm{m}^{2}$ & $34(54)$ & $7(41.2)$ & 0.349 \\
\hline Age (years) & $35(55.6)$ & $8(47.1)$ & 0.533 \\
\hline Isolated CABG & $60.41 \pm 4.01$ & $60.94 \pm 3.94$ & 0.63 \\
\hline Isolated valve surgery & $18(28.6)$ & $4(23.5)$ & 0.891 \\
\hline Combined CABG and valve & $26(41.3)$ & $8(47.1)$ & \\
\hline Diabetes mellitus & $19(30.2)$ & $5(29.4)$ & 0.489 \\
\hline Hypertension & $28(44.4)$ & $6(35.3)$ & 0.832 \\
\hline CPB(min) & $24(38.1)$ & $6(35.3)$ & 0.465 \\
\hline Levosimendan & $124.83 \pm 19.46$ & $128.76 \pm 20.28$ & 0.014 \\
\hline LVEF & $36(57.14)$ & $4 \rrbracket 23.5)$ & 0.137 \\
\hline HR & $35.42 \pm 3.02$ & $36.65 \pm 2.74$ & 0.179 \\
\hline Cl & $65.73 \pm 3.05$ & $64.59 \pm 3.20$ & 0.843 \\
\hline Results are expressed as mean \pm SD or number (\%) & $2.09 \pm 0.19$ & \\
\hline CABG, Coronary artery bypass grafting; BMI, body mass index; LVEF, left ventricular ejection fraction; & \\
\hline CPB: cardiopulmonary bypass; LCOS, Low cardiac output syndrome, ICU, Intensive Care Unit; HR, & \\
\hline Heart rates; Cl, Cardiac index. & & & \\
\hline
\end{tabular}

\section{Figures}




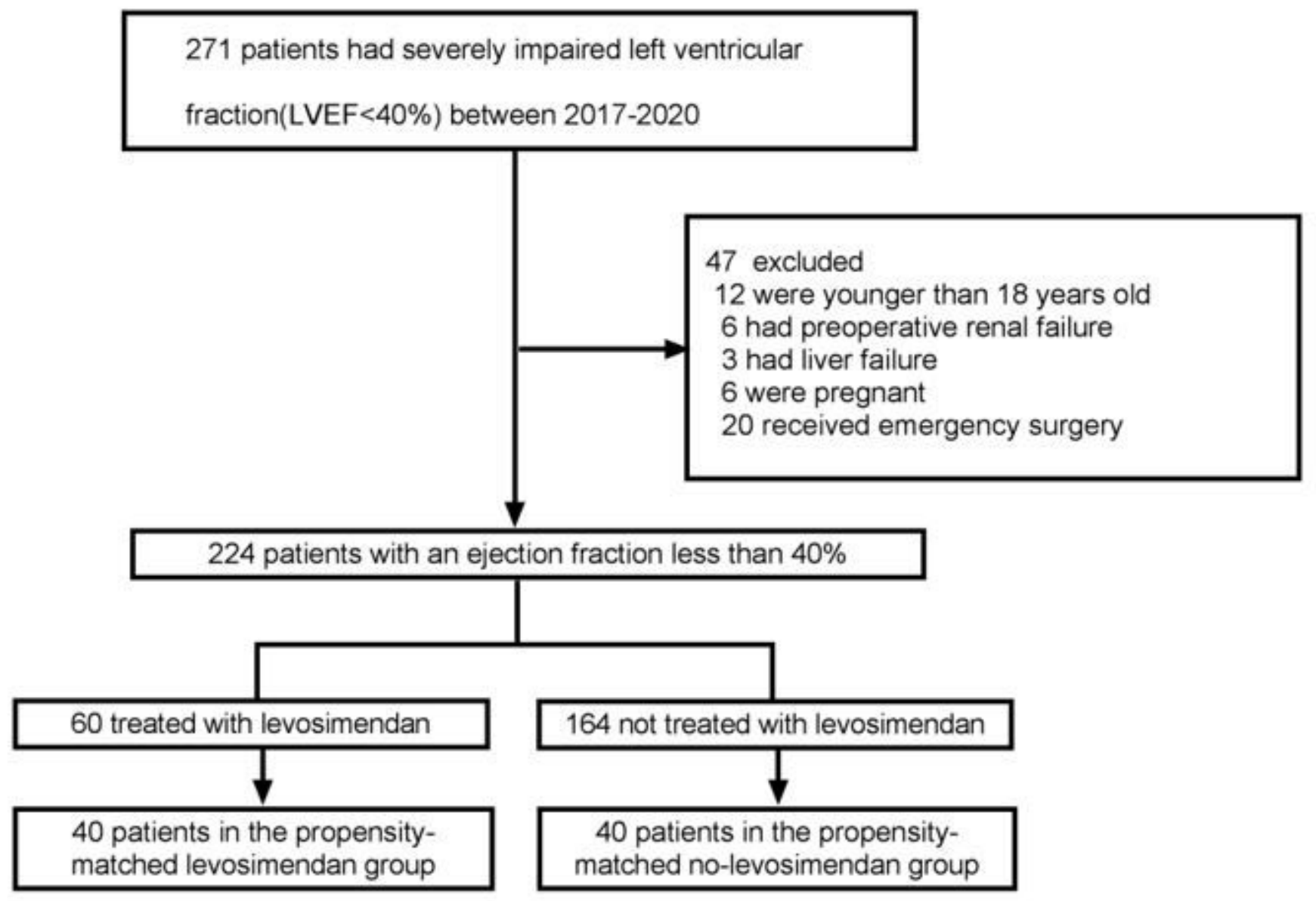

Figure 1

Selection of the study sample 

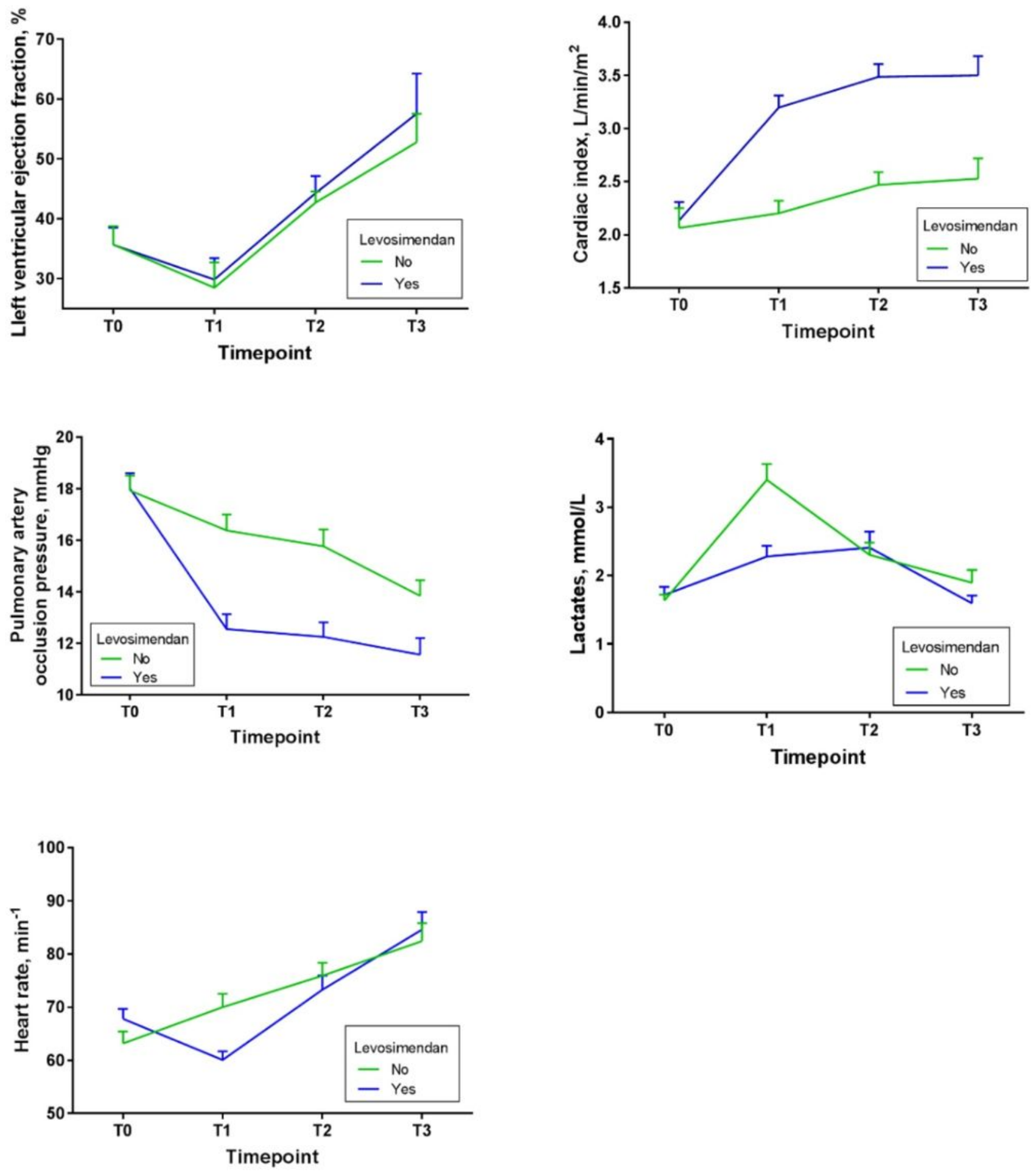

Figure 2

Baseline hemodynamic parameters and serum lactate concentration were comparable in both groups . 